\title{
The impact of participating in suicide research online
}

\section{Susanne Gibson, Zoë V R Boden, Outi Benson \& Sarah Brand}

\author{
For Suicide and Life Threatening Behavior
}

Published January 2014

\begin{abstract}
This study investigated the impact of participation in online mixed-methods suicide research. Participants, who described feeling suicidal, completed an 18-item questionnaire before and after taking part $(\mathrm{n}=103)$, and answered open-ended questions about participation $(\mathrm{n}=97)$. Overall, participation reduced negative experiences and had no effect on positive experiences. Feelings of calm increased, but participants felt less supported. Some participants did experience distress, but some also reported this distress to be manageable. Anonymously sharing experiences of suicidality was viewed as important, had therapeutic benefits, and engendered hopes for recovery. The findings suggest a need to ensure vulnerable participants in online studies are well supported, while protecting their anonymity.
\end{abstract}

\section{Introduction}

Over the last decade there has been substantial growth in the use of the internet in research, and at the same time, researchers have begun to respond to the call for more qualitative research on suicide, particularly with those who have first-hand experience of feeling suicidal or attempting suicide (Cutcliffe, 2003; Hjelmeland, 2010). However, while ethical research and the process of ethics review require an evidence base (Gibson, Benson \& Brand, 2012; Sharkey et al., 2011) there has been little systematic assessment of the impact of participating in suicide research, particularly qualitative research, on people who have experience of being suicidal (Biddle et al., 2013; Rivlin, Marzano, Hawton \& Fazel, 2012). Similarly, although there is a literature on the ethics of internet based research, including guidelines for researchers (Sharkey et al., 2011), there appears to be little available evidence on the impact of using the internet to gather data from vulnerable participants. 
Two recent studies assessing the impact of in-depth interviews on people with experience of suicide attempts or self-harm found that although some participants reported negative effects, overall, participants benefited from taking part in the research (Biddle et al., 2013; Rivlin et al., 2012). This is in line with previous findings on the impact of qualitative research with vulnerable participants, or on sensitive topics, which also suggest that the opportunity to talk in depth can be beneficial (Dyregrov, 2004; Hawton, Houston, Malmbergand \& Sinkin, 2003).

While Rivlin et al. (2012) and Biddle et al. (2013) provide evidence of the impact of taking part in face-toface interviews exploring the experience of suicide, the use of the internet in research raises additional concerns, as well as having potential advantages. The internet has opened up a range of possibilities for researchers, including treating information that is freely available on the internet as data, and using online tools to collect new data. In the first case there are ethical issues around the blurring of the distinction between what is public and what is private, and with regard to consent (Heilferty, 2011; Weeden, 2012). For example, although posts to a support forum for people with mental health difficulties might be easily accessed by researchers and others, it does not follow that it is ethical to use those posts as research data, much less to quote directly from them in publications. In the second case, while there are potential benefits to collecting data online for researchers, for example accessing a wider and larger sample of participants, and for participants, for example having the opportunity to describe their experiences anonymously and at a time and pace that suits them (Markham, 2011; Rodham \& Gavin, 2006; Marcell \& Falls, 2001), there are also risks involved. Not least, participants may be adversely affected by the research without the safety net of having another person present to notice and respond to their distress (British Psychological Society, 2007). Where the research addresses a sensitive topic with potentially vulnerable participants, this must be of particular concern.

In this paper we report on the impact of taking part in an online mixed-methods study investigating the experience of suicidal feelings. People who had experienced suicidal feelings, including some who had attempted suicide, completed a modified version of the Harkavy Asnis Suicide Scale (Harkavy-Friedman \& Asnis, 1989) and responded to the open-ended question 'What is it like to feel suicidal?'. To assess the impact of taking part in this study, participants completed a quantitative impact evaluation questionnaire at 
the beginning and end of the process, and gave qualitative feedback on their experience of taking part. It is on this data that we report here. While there were options to participate by telephone or post, the majority of participants completed the questionnaire online, either directly through the website, or in the case of 1 participant, by email. Since the purpose of this paper is to assess the impact of taking part in research online, the telephone and postal participants have been excluded from the analysis.

\section{Method}

\subsection{Participants}

There were 113 participants in this evaluation (of 124 who took part in the study). All completed the quantitative evaluation, and 97 completed the qualitative evaluation. There were 80 (70.8\%) female participants (two did not disclose gender). The age range was 16 to 67 years (mean=37.31, SD=12.96, one did not disclose age). The sample was recruited from across the UK, and the majority reported having a White British ethnicity ( $\mathrm{n}=95,84.1 \%) .92$ participants $(81.4 \%)$ reported one or more psychiatric diagnoses, with 40 participants having two diagnoses, 11 having three, and 1 having four diagnoses (see Table 1). [Insert Table

\section{1 here]}

\subsection{Materials and procedure}

To measure the impact of the suicidal feelings study participants were asked to complete one 7-point Likertstyle questionnaire before and after participation. The questionnaire was developed from the suicide literature and comprised 18 items describing experiences associated with suicidality and well-being (see Table 2), such as negativity about the self (Baumeister, 1990) or the future (Beck, Steer, Kovacs \& Garrison, 1985), and affects pertaining to interpersonal relating such as irritability (Graves \& Thomas, 1990) and loneliness (Hawton, Cole, O'Grady \& Osborn, 1982). Participants were asked to rate the extent to which they were experiencing each item at the present moment, from 1 (not at all) to 7 (very much so).

Participants were lastly requested to answer seven open-ended questions which asked about any upsetting aspects of the study, any feedback they had about the experience of participating, including good and bad aspects, improvements that could be made, problems they may have experienced, any positive or negative effects, and any benefits they may have experienced. 


\subsection{Ethics}

Ethical approval for the study was granted by the Joint UCL/UCLH Committee on the Ethics of Human Research (Committee Alpha) ref 10/H0715/5. Participants confirmed that they had read and understood the Participant Information sheet and gave their informed consent online (by clicking in the appropriate box). It was not possible for participants to progress with the online study until they had given their consent. The impact evaluation also served the purpose of monitoring participant well-being, both for the participants themselves and the researchers. Instructions on how to access 24-hour sources of support and (office hours) contact details for the research team were provided to all participants. Although participants were given the option to leave contact details, this was not a requirement and it was therefore possible to participate with complete anonymity.

\subsection{Data Analyses}

Quantitative data analyses were conducted using the Statistical Package for the Social Sciences (SPSS, Version 18.0 for Windows, SPSS Inc., 2009) with a significance threshold of $p<0.05$. The non-parametric withingroups Wilcoxon Signed Ranks Test was used to test differences before and after taking part in the study.

Qualitative data were analysed thematically (Braun \& Clarke, 2006). To enhance validity, this was carried out independently by two members of the research team (SG and ZB). After reading the transcripts thoroughly, data were coded inductively. Codes were then clustered into themes and subthemes according to their prevalence across the data-set, and with reference to potential theoretical interests. The aim was to summarise and describe the key thematic content of the data. A consensus discussion between SG and ZB led to the final thematic structure. Verbatim extracts were then chosen to best illustrate each theme.

\section{Results}

\subsection{Quantitative findings}

113 participants completed the quantitative self-report scale before and after taking part in the study. Median scores are reported as the data are not normally distributed. To gain an overview, composite median scores for the twelve negatively-valenced and suicidality items ('negative experiences'; irritated, pessimistic, confused, 
lonely, frustrated, self-critical, distressed, ashamed, urge to self-harm, suicidal intent, need to hide feelings, discomfort at discussing feelings) and six positively-valenced items ('positive experiences'; motivated, confident, interested, connected, supported) were generated. The median negative experiences score significantly decreased from 4.5 before the study to 4.0 after the study ( $\mathrm{z}=-3.524, \mathrm{p}<0.001)$. Fifty-six participants (49.6\%) showed a decrease in negative experiences, 37 (32.7\%) reported no change, and 20 (17.7\%) reported an increase in overall negative experiences. Median positive experiences scores showed no significant difference before (med=3.0) and after (med=3.0) the study ( $\mathrm{z}=-.290, \mathrm{p}>0.05)$. Forty-two participants (37.2\%) reported an increase in positive experiences, 37 (32.7\%) reported a decrease in positive experiences, and 34 (30.1\%) reported no change.

Looking item-by-item, Table 2 compares median ratings before and after taking part in the study. There were significant decreases in feelings of irritation/anger $(\mathrm{z}=-3.329, \mathrm{p}=0.001)$, pessimism/worry $(\mathrm{z}=-4.391, \mathrm{p}<0.001)$, confusion $(\mathrm{z}=-2.872, \mathrm{p}=0.004)$, frustration $(\mathrm{z}=-3.834, \mathrm{p}<0.001)$ and critical/disappointed with self $(\mathrm{z}=-2.765$, $\mathrm{p}=0.005)$. There were also significant decreases in urges to self-harm $(\mathrm{z}=-4.176, \mathrm{p}<0.001)$, suicidal intentions ( $\mathrm{z}=-3.963, \mathrm{p}<0.001)$, and discomfort with disclosing suicidal feelings $(\mathrm{z}=-2.366, \mathrm{p}=0.017)$. Of the positive experiences, there was a significant increase in feeling calm/relaxed $(\mathrm{z}=-2.448, \mathrm{p}=0.014)$, but a significant decrease in feeling supported $(\mathrm{z}=-2.614, \mathrm{p}=0.008)$. [Insert Table 2 here]

\subsection{Qualitative findings}

Four main themes categorise data from the 97 participants who completed the open-ended impact questions:

'Well-being and distress', 'The importance of being heard', 'Therapeutic impact' and 'Engendering hope'.

\subsubsection{Well-being and distress}

The first theme (sub-themes; 'Judging well-being', 'Managing distress' and 'Concern for others') shows participants reflecting on their recovery, and negotiating and managing feelings of distress.

Judging well-being: A number of participants felt that taking part encouraged them to reflect on their recovery process. They recognised that participating required a certain degree of mental strength, and that they needed 
to take responsibility for their well-being by making a careful judgement about their current mental health and their ability to cope with distressing feelings:

"Because overall I feel much better about myself and my life, I was able to complete the questions - even though I found doing so quite upsetting I don't think I would have been mentally strong enough to deal with this a year ago [...] distressing - but at a manageable level." (P52, Male).

"there are days when I wouldn't have done it as [it] would have been too triggering, but then the study warned about that and said to stop if [that] happened." (P78, Female)

Managing distress: Nearly a third of participants reported no upsetting aspects of taking part. However, of those who did experience distress, participation was described as "hard", "intense”, or "worrying”. However, distress and benefit were not understood as mutually exclusive: negative aspects were often framed as 'distressing, but...', suggesting that distress was perceived as manageable, and could be off-set against the more positive outcomes:

"I wouldn't say it is upsetting but it is challenging having to look back and describe feelings that are difficult for me." (P72, Male)

"It brought my feelings into focus and that was disturbing but helpful as well" (P5, Gender not specified)

It seemed inevitable to some participants that thinking about their suicidal feelings would cause distress. But again, participants asserted their ability to manage this distress, and expressed a confidence that it would pass quickly.

"Inevitably remembering is painful - it made me cry but I'm OK” (P77, Female)

Whilst for others, even though they experienced distress after participating, they believed this was not due to taking part:

"Even though I'm not in a good frame of mind at the moment I don't think answering the questions made me feel any worse and think it's great that research is being done." (P10, Female) 
Concern for others: A few participants worried that more vulnerable individuals might not be able to take such careful, informed decisions about their participation. The concern was that for these others taking part could be "dangerous":

"I would worry a little for young(er) people (under 25) reflecting upon and recounting their experiences of suicidal thoughts and feelings if they were isolated/alone and didn't have access to adequate support networks and/or if they were currently experiencing poor mental healt." (P3, Male)

Additionally two participants worried about the potentially triggering nature of the study, in particular the choice of language used in the suicidality measure ${ }^{\mathrm{i}}$ :

“'intend to kill myself" is a little blunt. It is a little harsh and perhaps could be worded differently." (P19, Female)

This theme suggests that although a third of participants reported no upsetting aspects of the study, for those who did experience distress this was connected to remembering painful experiences. However, for the majority, the level of distress felt manageable and for some it was viewed as an inevitable consequence of sharing their suicidal experiences. Most participants seemed to approach the study from an informed and reflective perspective, acknowledging where they were in their recovery, and their current ability to cope with their feelings. This was sometimes expressed as a concern that more vulnerable individuals could become overly distressed by participating. That is, there was a positioning of their own level of vulnerability in relation to others.

\subsubsection{The importance of being heard}

This theme (sub-themes: 'The struggle to be heard', 'Sharing without consequence' and 'Feeling heard, feeling supported') demonstrates the way in which participants' comments frequently focused on their ability to communicate with the researchers and the importance they placed on being heard. 
The struggle to be heard: For some participants the first challenge of being heard was articulating their suicidal feelings in ways that felt adequate. A few participants spoke of the difficulty connecting with feelings that felt distant:

"IIt was upsetting] just remembering how ill I have been in the past and it now seems as though that was a different person to me" (P94, Female)

Others struggled to verbalise feelings that felt too close or overwhelming:

"It was hard to describe in detail how feeling suicidal actually feels, but that might be because those feelings are fairly fresh..." (P73, Female)

However, a major concern for just under a quarter of participants was connected to the format of the suicidality measure ${ }^{\mathrm{ii}}$ which formed part of the study. Participants strongly expressed their frustration, and in some cases distress, at the limited number of responses available, especially when answering structured questions about how often they had certain experiences:

"I was extremely distressed by the two week/whole life comparisons, for the simple reason that the available choices were ludicrously poor. There are lots of things [i]n that list which I know I've felt upwards of twenty times in my life but rarely more than once in any given week [...] [It] may sound pedantic but this is the kind of thing that makes me panicky and anxious." (P4, Female)

This may be partly explained by participants' concern to answer questions honestly and accurately. If participants are to share these experiences (which are often painful), then it seems they want to ensure their experiences are being heard properly:

"I could not find a way to answer most of those questions accurately and feel I could not give an accurate impression of my experiences with suicidal thoughts. " (P8, Female)

As well as being upsetting and frustrating, occasionally the structured answers were perceived as excluding, only catering for individuals with particular types of experiences, or as challenging the validity of their experiences: 
"things don't always divide neatly into 'daily, 'once a week', etc. That was upsetting to think I was not being understood enough or that my depression was perhaps somehow 'wrong' when I couldn't think how to answer 'properly'." (P80, Female)

Sharing without consequence: For many participants, having the opportunity to share their experiences without consequence was important. The anonymity of the online format allowed participants to share without having to cope with others' reactions:

"It's good to be able to share without expecting or having to experience a response to those raw emotions." (P17, Female)

Some participants noted that they rarely or never had opportunities to share their suicidal feelings, and that this study made these feelings more permissible.

"It's almost impossible to be able to share thoughts, feelings, experiences relating to suicide with others and I find that it can build up inside. Taking part in a survey, provides a chance to share some of these experiences as if they were "normal'." (P75, Female)

"[It] made me think about my suicidal feelings in depth, as we don't really talk about them in depth to medical professionals, counsellors etc. It is very much a taboo subject." (P71, Female)

Feeling heard and feeling supported: Participants additionally described how taking part offered an experience of being listened to, making contact with someone, and more generally feeling supported in the knowledge that research is underway:

"It's good to know someone is listening." (P64, Male)

"It's a positive thought knowing that it's a sensitive subject that's being taken seriously and that the experiences of those affected are being listened to and acted on at some point in the future." (P70, Female)

This theme shows how the importance placed on being heard meant that a proportion of participants were frustrated by their perceived inability to articulate their experiences and by the rigidity of the suicidality measure. It was evident that describing their experiences accurately and honestly was paramount. However, 
for many participants the chance to speak anonymously, and without consequences and to know that someone would be listening to and taking their responses seriously, provided a feeling of support.

\subsubsection{Therapeutic Impact}

Included in this theme (subthemes: 'Catharsis' and 'Sense-making') are participants' accounts of beneficial aspects of taking part')

Catharsis: Just under a quarter of participants described a cathartic effect of taking part. They wrote about feelings of relief and release resulting from sharing their experiences:

"Writing about my suicidal feelings felt sort of relieving. I've never really discussed them in so much depth before. It was good to get the thoughts out of my head and into writing." (P59, Female)

"It is always good to 'let it out'! I feel a little lighter [...]" (P47, Female)

For some the process of writing reduced feelings of agitation:

"Writing down my feelings and experiences allowed me to express them, and feel more calm and relaxed." (P60, Male)

For several participants being in the study resulted in their feeling better, which often seemed to come as a surprise:

"I do feel more alert and positive for sharing this information and those feelings." (P79, Male)

"I actually feel slightly better!" (P93, Female)

Sense-making: A second, overlapping effect of participating was the increased insight and self-awareness that some participants experienced. This seemed to be particularly connected to the process of formulating responses and verbalising feelings. Through structuring their experiences in language, participants gained greater self-understanding:

"It made things clearer in my head - it was useful to have to think really hard about the question and then organise my thoughts. It sort of helped me realise what I was thinking” (P22, Female) 
For one participant this resulted in feeling better able to cope with the feelings:

"Having to write about feeling suicidal has made me examine my feelings more closely. Whilst this might upset some people it has enabled me to face my feelings and like facing my fears it has made them easier to bear. I feel like I can cope with them better." (P81, Female)

This theme illuminates the therapeutic impact of taking part; firstly many had a cathartic experience, and for some this led to a reduction in negative feelings or an increase in positive feelings. Secondly, participants described how structuring their experiences in order to answer the questions gave them greater insight and allowed them to use their sense-making capacities to become more aware of their thoughts and feelings related to suicide.

\subsubsection{Engendering Hope}

The final theme (subthemes: 'Hoping to help', and 'Hopes for recovery') explores participants' expressions of hope for others and themselves)

Hoping to help: Just over a sixth of participants explicitly expressed a hope that by sharing their story they could be of help to others. Other participants expressed a desire to be "useful", to do something "productive", to make a contribution, or to be part of something "good":

"[Benefits include] the hope that the research will make a difference and I will have played a part in it." (P76, Female)

"It felt useful as my experience may help others - largely [I] feel useless." (P61, Female)

In particular, some participants expressed a hope that their suffering would not be in vain, and that through their participation others might benefit:

"I do feel slightly better in thinking that my life of *\&\% may not be for nothing, hopefully some of the information I have locked in my head may help people in the future to not have to live this nightmare." (P13, Male) 
Some participants expressed the hope that their responses may serve to educate others and to contribute to the understanding of suicidal experiences more generally, for example by countering prejudices:

"I hope it may prove useful for me and others to talk. It may lead to better understanding and over time changes of attitude." (P79, Male)

Hopes for recovery: Whilst the 'hope to help' centres on benefits for others, a smaller number of participants expressed hopes for themselves and their recovery. For a few, this meant gaining the motivation to seek help: "surprisingly I feel a little more motivated. I'm thinking of getting help now" (P20, Male)

"It has also given me a little confidence that I might need to trust a professional to help me instead of trying to berate myself for having these feelings." (P80, Female)

For a few participants, writing about their experiences encouraged them to explore ways to improve communication with their healthcare professionals or family:

"I'm wondering whether to print [my responses] out and give [them] to [the] GP or nurse. If it makes any sense in [the] broad light of day, it might help us all understand more and be able to work out useful approaches/help." (P78, Female)

This theme illustrates how participants' expressions of hope mainly centre on helping others, whether as a hope to contribute to something worthwhile, or a hope that their experiences can have some purpose. For a few, the study also provided motivation to seek help for themselves or to open up a little more to family or healthcare professionals. Although relatively few participants described these hopes for recovery, clearly this indicates a significant outcome for those individuals.

\section{Discussion}

Qualitative suicide research and online research are both important 'growth' areas and the findings from this study contribute to a small but developing body of evidence regarding the impact on participants (Biddle et al., 2013; Rivlin et al., 2012; Sharkey et al., 2011; Hawton et al., 2003). 
Overall, the results appear to be in line with previous studies of qualitative research on sensitive topics and/or with vulnerable participants, including those with experience of suicide (Biddle et al., 2013; Rivlin et al., 2012; Dyregov, 2004; Hawton et al., 2003). That is, the data from the study suggest that participants are likely to benefit from taking part. On the quantitative measure, while some participants felt worse or the same, overall there was a significant reduction in negative experiences. However, that participants felt less supported requires further exploration. Although this was not explicitly addressed in the qualitative data provided by the participants, it is worth hypothesising on why this might have been the case. One possibility is that this is specifically related to taking part in online research where there is no direct contact with the researcher.

The negative impact of the absence of the researcher is perhaps exemplified in one aspect of the experience that some participants found upsetting; that is the difficulty completing the measure of suicidality because it didn't allow a sufficient range of options. Some of this negative impact might have been mitigated through the presence of a researcher able to support decision-making, or just to acknowledge the participant's dissatisfaction. In face-to-face research, both quantitative and especially qualitative, there tends to be an inbuilt element of debriefing, checking understanding and reassurance, suggesting a need to find ways to incorporate this aspect of the process into online research. For example, in addition to giving participants an opportunity to reflect on the experience of taking part in their own words, this might be achieved by providing contact details for a named researcher (Sharkey et al., 2011), or having a tick box for participants who wish to receive a personalised acknowledgement.

On the whole, however, while some participants reported upsetting aspects of the study, these were often balanced by benefits or were accepted as inevitable when taking part in a study on feeling suicidal, with some participants distinguishing between negative feelings associated with engaging in the research, and distressing feelings associated with being suicidal. Thus it appears that participants felt able to assess and manage their distress, and to reflect on this process; that is, they articulated a capacity to make an informed decision about taking part (Lakeman \& Fitzgerald, 2009a; Lakeman \& Fitzgerald, 2009b).

Further, while it has been claimed that talking about experiences of being suicidal can be therapeutic (Biddle et al., 2013; Smith, Poindexter \& Cukrowicz, 2010), this study suggests that writing online about those 
experiences can be similarly beneficial and might even offer participants a special opportunity to articulate and structure their thoughts. Thus by providing a "chrono-malleable" environment in which to reflect on and edit responses (Markham, 2011) online research can combine a greater degree of control over the process of responding, with the knowledge that someone will read and take what has been written seriously. Indeed, for some, there was a sense that the product of the process could be useful to communicate something of clinical importance: three participants commented that they were considering taking what they had written to share with a therapist or healthcare provider.

The experience of participating anonymously afforded by taking part online was also beneficial insofar as it allowed participants to write about their experiences without any consequences in terms of others' reactions. It is worth noting that the median score on the item 'Feel a need to hide my experience of suicidal feelings from those close to me' was a constant 6 (with 7 being 'very much so') for this group of people. Online anonymous research might therefore succeed in reaching groups of people who are not willing to risk a faceto-face encounter, or who are not able to share their experiences with significant others, and in doing so benefit both the participant and the research (Rodham \& Gavin, 2006).

\subsection{Strengths and limitations of the study}

The analysis presented here assesses the immediate impact of research participation. While this can be considered a strength of the study, it would have been informative to collect additional data on the longerterm impact on participants.

We do not have data on the impact of participation on those who dropped out of the study part way through and it is possible that the impact of taking part was a reason for non-completion.

Some participants $(n=16)$ did not complete the open-ended questions about their experience of taking part and some others gave very short or one-word answers. Having a researcher present might have elicited a greater response rate and provided the opportunity to probe for more information or to clarify the meaning of an expression (Rivlin et al., 2012). The absence of the researcher also meant that they were not able to access the non-verbal information available in a face-to-face or telephone interview, such as body language or tone of voice (Rodham \& Gavin, 2006). However, the online method is more 'participant-led', in the 
sense that participants have more control over their responses without the influence of having a researcher present. This helps to shift the power from researcher to participant, and enhances validity by minimising bias (Marcell \& Falls, 2001).

\subsection{Conclusions}

Attending to the experience of taking part in research is vital for participant care. In addition, robust evidence is required to inform ethical practice and ethics review, including finding ways for the voice of the participant to be heard in the process of ethics decision-making (Gibson et al., 2012).

The results of this study suggest that it is possible to undertake qualitative/mixed-methods suicide research online in a way that safeguards the well-being of participants, and that participants who have experienced suicidal feelings are able to make an informed decision to take part.

In online research on sensitive topics and/or with vulnerable participants, particular attention should be paid to supporting participants, both during and after taking part. This includes developing ways to facilitate contact between participant and researcher, where this is desired. At the same time, the opportunity for people to have their voices heard while remaining completely anonymous should be retained within the scope of suicide research. Finding ways to provide support under conditions of anonymity is therefore similarly important and presents an additional challenge.

\section{References}

Baumeister, R. F. (1990). Suicide as escape from self. Psychological Review, 97, 90-113.

Beck, A.T., Steer, R. A., Kovacs, M., \& Garrison, B. (1985). Hopelessness and eventual suicide: A 10-year prospective study of patients with suicidal ideation. American Journal of Psychiatry, 142, 680-686

Biddle, L., Cooper, J., Owen-Smith, A., Klineberg, E., Bennewith, O., Hawton, K., Kapur, N., Donovan, J., \& Gunnell, D. (2013). Qualitative interviewing with vulnerable populations: individuals' experiences of participating in suicide and self-harm based research. Journal of Affective Disorders, 145, 356-362 
Braun, V. \& Clarke, V. (2006). Using thematic analysis in psychology. Qualitative Research in Psychology, 3, 77-101.

British Psychological Society (2007). Report of the working party on conducting research on the internet: Guidelines for ethical practice in psychological research online. Available from http://www.bps.org.uk/sites/default/files/documents/conducting_research_on_the_internetguidelines_for_ethical_practice_in_psychological_research_online.pdf (accessed 03/04/2013)

Cutcliffe, J.R. (2003). Research endeavours into suicide: a need to shift the emphasis. Mental Health Nursing, 12, 92-99.

Dyregrov, K. (2004). Bereaved parents' experience of research participation. Social Science \& Medicine, $\mathbf{5 8}$, 391-400.

Gibson, S., Benson, O., \& Brand, S.L. (2012). Talking about suicide: Confidentiality and anonymity in qualitative research. Journal of Nursing Ethics, 20, 18-29.

Graves, P. L. \& Thomas, C. B. (1990). Habits of nervous tension and suicide. Suicide and Life-threatening Behaviour, 21, 91-105.

Harkavy Friedman, J.M., \& Asnis, G.M. (1989). Assessment of suicidal behavior: A new instrument. Psychiatric Annals, 19, 382-387.

Hawton, K., Cole, D., O’Grady, J., \& Osborn, M. (1982). Motivational aspects of deliberate self-poisoning in adolescents. British Journal of Psychiatry, 148, 586-591.

Hawton, K., Houston, K., Malmbergand, A., \& Sinkin, S. 2003. Psychological autopsy interviews in suicide research: the reactions of informants. Archives of Suicide Research, 7, 73-82.

Heilferty, C.M. (2011). Ethical considerations in the study of online illness narratives: A qualitative review. Journal of Advanced Nursing, 67, 945-953.

Hjelmeland, H. \& Knizek, B.L. (2010). Why we need qualitative research in suicidology. Suicide and LifeThreatening Behaviour, 40, 74-80. 
Lakeman, R. \& Fitzgerald, M. (2009a). Ethical suicide research: A survey of researchers. International Journal of Mental Health Nursing, 18, 10-17.

Lakeman, R. \& Fitzgerald, M. (2009b). The ethics of suicide research: The views of ethics committee members. Crisis, 30, 13-19.

Marcell, M.M. \& Falls, A.L., (2001). Online data collection with special populations over the world wide web. Down Syndrome Research and Practice, 7, 106-123.

Markham, A.N., 2011. Internet research. In D. Silverman (Ed.), Qualitative Research, $3^{\text {rd }}$ Edition (pp. 111127). London: Sage.

Rivlin, A., Marzano, L., Hawton, K., \& Fazel, S. (2012). Impact on prisoners of participating in research interviews related to near-lethal suicide attempts. Journal of Affective Disorders, 136, 54-62.

Rodham, K. \& Gavin, J., (2006). The ethics of using the internet to collect qualitative research data. Research Ethics Review, 2, 92-97.

Sharkey, S., Jones, R., Smithson, J., Hewis, E., Emmens, T., Ford, T., \& Owens, C. (2011). Ethical practice in internet research involving vulnerable people. Journal of Medical Ethics, 37, 752-758.

Smith, P., Poindexter, E., \& Cukrowicz, K. (2010). The effect of participating in suicide research: Does participating in a research protocol on suicide and psychiatric symptoms increase suicide ideation and attempts? Suicide and Life-Threatening Behaviour, 40, 535-543.

SPSS Inc., (2009). PASW Statistics for Windows, Version 18.0. Chicago: SPSS Inc.

Weeden, M.R. (2012). Ethics and on-line research methodology. Journal of Social Work Values and Ethics, 9, 40-51. 


\section{Tables:}

Table 1: Participants' self-reported diagnoses (*due to reporting multiple diagnoses, percentages do not total 100).

\begin{tabular}{|l|l|}
\hline Diagnosis & \%* (n) \\
\hline Depression & $52.2 \%(59)$ \\
\hline Anxiety & $18.6 \%(21)$ \\
\hline Borderline Personality Disorder & $20.3 \%(23)$ \\
\hline Bipolar Disorder (Type I) & $8.0 \%(9)$ \\
\hline Post Traumatic Stress Disorder & $5.3 \%(6)$ \\
\hline Schizophrenia & $5.3 \%(6)$ \\
\hline Eating Disorders (various) & $2.7 \%(3)$ \\
\hline Obsessive Compulsive Disorder & $2.7 \%(3)$ \\
\hline Psychosis (not specified) & $1.8 \%(2)$ \\
\hline Schizoaffective Disorder & $1.8 \%(2)$ \\
\hline Agoraphobia & $0.9 \%(1)$ \\
\hline Asperger Syndrome & $0.9 \%(1)$ \\
\hline Attention Deficit Hyperactivity Disorder & $0.9 \%(1)$ \\
\hline Bipolar Disorder (Type II) & $0.9 \%(1)$ \\
\hline Body Dysmorphic Disorder & $0.9 \%(1)$ \\
\hline Dissociative disorders (various) & $1.8 \%(2)$ \\
\hline Mood disorder (not specified) & $0.9 \%(1)$ \\
\hline Other personality disorder & $0.9 \%(1)$ \\
\hline Stress & $0.9 \%(1)$ \\
\hline No diagnosis received & $18.6 \%(21)$ \\
\hline & \\
\hline
\end{tabular}


Table 2. Item-by-item comparison of experiences before and after the study $\left(* p<0.05,{ }^{* *} p \leq 0.001\right.$ two-tailed)

\begin{tabular}{|c|c|c|c|c|c|c|c|}
\hline Self-report questionnaire item & $\begin{array}{l}\text { Median } \\
\text { rating } \\
\text { before }\end{array}$ & $\begin{array}{l}\text { Median } \\
\text { rating } \\
\text { after }\end{array}$ & $N$ & Z score & $\begin{array}{l}\%(n) \text { score } \\
\text { increase }\end{array}$ & $\begin{array}{l}\%(n) \text { score } \\
\text { decrease }\end{array}$ & $\begin{array}{l}\%(n) \text { score } \\
\text { unchanged }\end{array}$ \\
\hline Feel irritated or angry & 4.0 & 3.0 & 113 & $-3.329 * *$ & $11.5 \%(13)$ & $39.8 \%(45)$ & $48.7 \%(55)$ \\
\hline $\begin{array}{l}\text { Have pessimistic or worried } \\
\text { thoughts about the future }\end{array}$ & 6.0 & 5.0 & 112 & $-4.391 * *$ & $16.1 \%(18)$ & $42.9 \%(48)$ & $41.1 \%(46)$ \\
\hline Feel confused about my feelings & 5.0 & 4.0 & 111 & $-2.872^{*}$ & $21.6 \%(24)$ & $39.6 \%(44)$ & $38.7 \%(43)$ \\
\hline Feel lonely & 5.0 & 5.0 & 113 & -.810 & $22.1 \%(25)$ & $28.3 \%(32)$ & $49.6 \%(56)$ \\
\hline Feel frustrated & 5.0 & 4.0 & 111 & $-3.834 * *$ & $17.1 \%(19)$ & $45.0 \%(50)$ & $37.8 \%(42)$ \\
\hline $\begin{array}{l}\text { Feel critical of / disappointed with } \\
\text { myself }\end{array}$ & 6.0 & 5.0 & 109 & $-2.765^{*}$ & $18.3 \%(20)$ & $40.4 \%(44)$ & $41.3 \%(45)$ \\
\hline Feel an urge to harm myself & 3.5 & 3.0 & 113 & $-4.176 * *$ & $14.2 \%(16)$ & $39.8 \%(45)$ & $46.0 \%(52)$ \\
\hline Intend to kill myself & 2.0 & 1.5 & 113 & $-3.963 * *$ & $10.6 \%(12)$ & $33.6 \%(38)$ & $55.8 \%(63)$ \\
\hline Feel distressed & 4.0 & 4.0 & 111 & -1.912 & $24.3 \%(27)$ & $36.9 \%(41)$ & $38.7 \%(43)$ \\
\hline $\begin{array}{l}\text { Feel shame about my suicidal } \\
\text { feelings (past or present) }\end{array}$ & 5.0 & 5.0 & 112 & -.223 & $25.0 \%(28)$ & $23.2 \%(26)$ & $51.8 \%(58)$ \\
\hline $\begin{array}{l}\text { Feel a need to hide my experience } \\
\text { of suicidal feelings from those } \\
\text { close to me }\end{array}$ & 6.0 & 6.0 & 112 & -.764 & $17.9 \%(20)$ & $24.1 \%(27)$ & $58.0 \%(65)$ \\
\hline $\begin{array}{l}\text { Feel discomfort at the thought of } \\
\text { discussing my suicidal feelings with } \\
\text { another person }\end{array}$ & 4.0 & 3.5 & 111 & $-2.366^{*}$ & $18.9 \%(21)$ & $30.6 \%(34)$ & $50.5 \%(56)$ \\
\hline Feel motivated & 3.0 & 4.0 & 113 & -.801 & $32.7 \%(37)$ & $25.7 \%(29)$ & $41.6 \%(47)$ \\
\hline Feel confident & 2.0 & 2.5 & 113 & -1.018 & $35.4 \%(40)$ & $23.9 \%(27)$ & $40.7 \%(46)$ \\
\hline $\begin{array}{l}\text { Feel interested and engaged with } \\
\text { daily life }\end{array}$ & 3.0 & 3.0 & 112 & -1.172 & $22.3 \%(25)$ & $30.4 \%(34)$ & $47.3 \%(53)$ \\
\hline Feel calm / relaxed & 3.0 & 3.0 & 112 & $-2.448^{*}$ & $46.4 \%(52)$ & $26.8 \%(30)$ & $26.8 \%(30)$ \\
\hline Feel able to connect with others & 3.0 & 3.0 & 112 & -1.086 & $25.0 \%(28)$ & $33.9 \%(38)$ & $41.1 \%(46)$ \\
\hline Feel supported & 4.0 & 3.0 & 108 & $-2.614^{*}$ & $18.5 \%(20)$ & $36.1 \%(39)$ & $45.4 \%(49)$ \\
\hline
\end{tabular}

i \& ii A modified version of the Harkavy Asnis Suicide Scale (Harkavy-Friedman and Asnis, 1989) was included in the study 